Forschende

Komplementärmedizin und

Klassische Naturheilkunde
Editorial

Forsch Komplementärmed Klass Naturheilkd 2005;12:132-133

DOI: $10.1159 / 000085539$

\title{
Den Stier bei den Hörnern packen!
}

\author{
Rainer Lüdtke \\ Karl und Veronica Carstens-Stiftung, Essen, Deutschland
}

Komplementärmedizin ist ein Wachstumsmarkt, nicht nur in Amerika, sondern auch in Deutschland und der Schweiz: 70\% der Frauen und 54\% der Männer in Deutschland haben schon einmal eine komplementärmedizinische Therapie in Anspruch genommen. Etwa die Hälfte aller Patienten würde es begrüssen, wenn ihre Ärzte ihnen entsprechende Therapien häufiger verschreiben oder ihnen Informationen hierzu anbieten würden [1]. Auch bei schweren chronischen Erkrankungen wie Morbus Crohn oder Colitis ulcerosa beträgt der Anteil der Patienten, die bereits Homöopathie, Phytotherapie oder Traditionelle Chinesische Medizin (TCM) für ihre Krankheit ausprobiert haben, etwa 50\%. Dieses belegen zwei nahezu zeitgleich erschienene Studien [2, 3].

Dem gestiegenen Interesse der Patienten steht eine eher abwartende Haltung der Universitäten gegenüber. Wie Brinkhaus und Koautoren [4] in diesem Heft belegen, sind mehr als ein Drittel der Entscheidungsträger in den medizinischen Hochschulen Deutschlands gegenüber der Komplementärmedizin aufgeschlossen. Allerdings lehnen auch fast genauso viele Institutsleiter die Komplementärmedizin ab, insbesondere Homöopathie und TCM, just die Methoden, die von den Patienten bevorzugt werden.

Hier besteht eine Lücke! Eine Lücke, die letztendlich nur durch Forschungsaktivitäten geschlossen werden kann. Wie Jonas [5] in dieser Ausgabe der Forschenden KomplemenTÄRMEDIZIN analysiert, ignoriert die derzeitige Forschungsförderung aber genau die strittigen Punkte: mehr als $80 \%$ der entsprechenden Gelder gehen in Bereiche, die bereits im Sinne der konventionellen Medizin akzeptiert sind (Identifikation der wirksamen Bestandteile von Pflanzenextrakten, Anwendung bereits etablierter Modelle zur Verhaltensände- rung, ...). Die kontroversen Knackpunkte, an denen sich konventionelle und komplementäre Ansätze fundamental unterscheiden (holistische Sichtweise auf Patienten, Stellenwert der Spiritualität, energetische Informationsübertragung, Ausnutzen von Interaktionseffekten, ...), werden dagegen gerade mal mit $2 \%$ der Gelder erforscht.

Aber gerade diese Konzepte sind es, die als Hauptverkaufsargumente für die Komplementärmedizin dienen und die $\mathrm{Pa}$ tienten attraktiv finden. Man kann also Jonas nur zustimmen und fordern, den Stier bei den Hörnern zu packen! Es geht um die Kernannahmen der Komplementärmedizin, die erforscht werden müssen! Dabei geht es nur sekundär um die Etablierung der Komplementärmedizin an den Universitäten. Vielmehr steht hierbei im Vordergrund, die Grundlagen der verschiedenen komplementären Ansätze wissenschaftlich so zu untermauern, dass hierauf ein «Evidence House», wie Jonas es nennt, errichtet werden kann. Ein solches Haus, von Jonas im Gegensatz zur evidenzbasierten Hierarchie der konventionellen Medizin konzipiert, kann nur getragen werden von festen Fundamenten. Das heisst: auf einer festen Basis werden sich die verschiedenen komplementärmedizinischen Ansätze nicht nur nach aussen rechtfertigen können, sondern auch intern weiter entwickeln können.

Ähnlich wie früher zum potenziellen Wirkmechanismus der Homöopathie [6] oder in diesem Heft zur Gültigkeit des Simile-Prinzips [7] wird sich die Forschende KomplemenTÄRMEDIZIN daher auch in Zukunft den Grundannahmen komplementärer Medizinkonzepte widmen. Ohne dabei jedoch den eigentlichen Zweck der Forschung aus den Augen zu verlieren: eine optimale Versorgung der Patienten.

\begin{tabular}{llll}
\hline KARGER & ( 2005 S. Karger GmbH, Freiburg & & Dipl.-Stat. Rainer Lüdtke \\
Fax +49 7614520714 & Accessible online at: & Karl und Veronica Carstens-Stiftung \\
E-mail Information@Karger.de & www.karger.com/fkm & Am Deimelsberg 36 \\
www.karger.com & & 45276 Essen, Deutschland \\
& & Tel. +49 2015630516, Fax 5630530 \\
& & E-mail r.luedtke@carstens-stiftung.de
\end{tabular}




\section{Literatur}

1 Härtel U, Volger E: Inanspruchnahme und Akzep$\tan z$ klassischer Naturheilverfahren und alternativer Heilmethoden in Deutschland - Ergebnisse einer repräsentativen Bevölkerungsstudie. Forsch Komplementämed Klass Naturheilkd 2004;11(6): 327-34.

2 Ausfeld-Hafter B, Hoffmann S, Seibold F, Quattropani C, Heer P, Straumann A: Stellenwert der Komplementärmedizin bei M.-Crohn- und Colitisulcerosa-Patienten: Eine Fragebogenerhebung. Forsch Komplementärmed Klass Naturheilkd 2005;12:134-138.
3 Langhorst J, Anthonisen IB, Steder-Neukamm U, Lüdtke R, Spahn G, Michalsen A, Dobos GJ: Amount of systemic steroid medication is a strong predictor for the use of complementary and alternative medicine in patients with inflammatory bowel disease: results from a German national survey. Inflamm Bowel Dis 2005;11(3):287-95.

4 Brinkhaus B, Joos S, Lindner M, Kohnen R, Witt C, Willich SN, Hahn EG: Integration of complementary and alternative medicine into German medical school curricula - contradictions between the opinions of decision makers and the status quo. Forsch Komplementärmed Klass Naturheilkd 2005;12:139_ 143.
5 Jonas WB: Building an evidence house: Challenges and solutions to research in complementary and alternative medicine. Forsch Komplementärmed Klass Naturheilkd 2005;12:159-167.

6 Walach H: Entanglement model of homeopathy as an example of generalized entanglement predicted by weak quantum theory. Forsch Komplementärmed Klass Naturheilkd 2003;10(4):192-200.

7 Brien S, Lewith G: Assessing homeopathic proving using questionnaire methodology: Consideration and implications for future studies. Forsch Komplementärmed Klass Naturheilkd 2005;12:152-158. 